\title{
Plasma Membrane Ganglioside Sialidase Regulates Axonal Growth and Regeneration in Hippocampal Neurons in Culture
}

\author{
Jose Abad Rodriguez,, ${ }^{2,3}$ Eugenia Piddini, ${ }^{3}$ Takafumi Hasegawa, ${ }^{1}$ Taeko Miyagi, ${ }^{1}$ and Carlos G. Dotti ${ }^{2,3}$ \\ ${ }_{1}^{1}$ Miyagi Prefectural Cancer Center, Division of Biochemistry, Medeshima-Shiode, Natori, Miyagi, 981-1293 Japan, \\ ${ }^{2}$ Cavalieri Ottolenghi Scientific Institute, Universita degli Studi di Torino, A. O. San Luigi Gonzaga, Regione Gonzole 10, \\ 10043 Orbassano (TO), Italy, and ${ }^{3}$ Cell Biology and Biophysics Program, European Molecular Biology Laboratory, 69012 \\ Heidelberg, Germany
}

It has been long recognized that the ganglioside GM1 plays a role in axonal growth and neuronal differentiation. However, the involvement of plasma membrane GM1 has been difficult to elucidate. This is possible now thanks to the recent cloning of plasma membrane ganglioside sialidase (PMGS), the enzyme responsible for the localized hydrolysis of oligosialogangliosides into GM1. In this work we show that PMGS mRNA and protein levels are high at early developmental stages of the hippocampus and low in adulthood both in vivo and in vitro. We also demonstrate that inhibition of PMGS activity blocks axonal elongation, whereas the increase in PMGS activity dramatically enhances axon growth and accelerates the polarization of cytoskeletal proteins. Finally, we show that axotomy close to the cell body in PMGS overexpressing neurons results in the regrowth of the original axon instead of randomly, as is the case in control neurons. In all, these results imply that PMGS activity through the modulation of GM1 surface levels is an important component of the machinery controlling axonal growth. We hypothesize that increasing PMGS activity in the adult nervous system may be useful to improve regeneration after nerve damage.

Key words: plasma membrane sialidase; ganglioside; hippocampal neuron; axonal growth; axonal regeneration
Axonal growth in vivo is a complex event in which the mechanisms of elongation and guidance must coordinately interplay. Despite the many years of intense work, only recently has a general picture emerged of how elongation and guidance are regulated at the molecular level (for review, see Tessier-Lavigne and Goodman, 1996; Suter and Forscher, 1998; Quinn et al., 1999; Andersen and Bi, 2000; Bradke and Dotti, 2000). Regarding the early event of axon formation, much of our current knowledge comes from studies in neuronal cells growing in culture. Thus, we know that axonal elongation requires the addition of new membrane at the tip of the growing neurite, an increase in the dynamics of actin fibers at the same site, an increase in the stability of the trailing microtubules in the shaft, and constant changes in the binding properties of membrane proteins and membrane lipids to the extracellular matrix. In this last event, gangliosides seem to play a major role.

Gangliosides are sialic acid-containing glycosphingolipids particularly enriched in the nervous tissue. Like most gangliosides, the monosialoganglioside GM1 was shown to modulate the growth and differentiation of neuroblastoma cells (Masco et al., 1991; Wu and Ledeen, 1991). In cultured neurons, ganglioside biosynthesis is essential for axonogenesis, axonal growth (Harel and Futerman, 1993; Schwarz et al., 1995), and synaptogenesis (Inokuchi et al., 1997). Also, addition of exogenous GM1 promotes differentiation and neurite outgrowth of neuronal cells in cultures (Facci et al., 1984; Skaper et al., 1985; Leeden, 1989), and

\footnotetext{
Received June 11, 2001; revised Aug. 6, 2001; accepted Aug. 24, 2001.

We thank Drs. Frank Bradke, Lola Ledesma, Jorge Santos Da Silva, and Beatriz Gil for scientific advice and discussions, and Bianca Hellias and Etienne Cassin for the preparation and maintenance of the hippocampal neurons.

Correspondence should be addressed to Cavalieri Ottolenghi, Scientific Institute, Universita degli Studi di Torino, A. O. San Luigi Gonzaga, Regione Gonzole 10, 10043 Orbassano (TO), Italy. E-mail: carlos.dotti@unito.it

Copyright (ㄷ) 2001 Society for Neuroscience $\quad 0270-6474 / 01 / 218387-09 \$ 15.00 / 0$
}

the possible potentiation of effect of some neurotrophic factors has been suggested (Katoh-Semba et al., 1984). More recently, the influence of gangliosides on the action of neurotrophins has been documented. In particular, GM1 interacts with the NGF receptors TrkA (Mutoh et al., 1993, 1995; Ferrari et al., 1995; Rabin and Mocchetti, 1995) and p75 NGFR (Panni et al., 1998), as well as with the BDNF receptor TrkB (Pitto et al., 1998). The use of GM1 binding reagents, like the subunit B of cholera toxin (Chtx-B), and anti-GM1 antibodies also supports a role for GM1 in axonal growth. Thus, Chtx-B was shown to induce differentiation in N18 neuroblastoma cells (Masco et al., 1991) and to block neuraminidase-induced neuritogenesis. Similarly, antibodies to GM1 have been shown to inhibit the NGF-induced sprouting of chick embryonic dorsal root ganglion cells (Schwartz and Spirman, 1982) and conditioned media-stimulated growth in sensory ganglion cells (Spoerri et al., 1988). Additionally, Wu and Ledeen, (1994) demonstrated that anti-GM1 antibodies inhibit the differentiation of Neuro2a cells stimulated by different agents. All of this evidence is clearly indicative of GM1 playing a role in axonal growth, but most of the experimentation has depended on the addition of exogenous GM1 or GM1 blockers to cultures of neuron-like cell lines. These facts prompted us to investigate the physiological role of the endogenous plasma membrane GM1 in primary neurons. For that purpose we have modified the levels and activity of plasma membrane ganglioside sialidase (PMGS) in hippocampal neurons in primary culture. PMGS is unique in specifically hydrolyzing oligosialogangliosides to produce GM1 in plasma membrane (Miyagi et al., 1999; Wada et al., 1999; Hasegawa et al., 2000).

\section{MATERIALS AND METHODS}

Isolation of $m R N A$ and $R T-P C R$. Poly $\left(\mathrm{A}^{+}\right)$RNA was affinity purified with the QuickPrep Micro mRNA purification kit (Amersham Pharmacia 
Biotech, Uppsala, Sweden) from isolated hippocampi [both from embryonic day (E) 15 embryos and from 2-month-old adult mice].

cDNA synthesis was performed using $\sim 10 \mathrm{ng}$ of mRNA and the Moloney murine leukemia virus reverse transcriptase (Advantage RTfor-PCR kit, Clontech Laboratories, Palo Alto, CA). Specific primers were designed to monitor membrane sialidase expression by PCR amplification (forward: TCAATTGGCAAAGGTGCTCCCGCTACTCAG; reverse: GGGCTGGTACAGTCTTCACAGCTCAGGACC). The PCR reactions contained $\sim 1 \mathrm{ng}$ cDNA, $1 \mu \mathrm{l}$ of a $10 \mathrm{~mm}$ solution of each dNTP, 1 U AmpliTaq DNA polymerase (Perkin-Elmer, Norwalk, CT), 20 pmol of each primer, in $1 \times$ Perkin-Elmer PCR Buffer (containing 1.5 $\mathrm{mM} \mathrm{MgCl}_{2}$ ) in a total volume of $50 \mu \mathrm{l}$. The PCR amplification consisted of 33 cycles of (1) $10 \mathrm{sec}$ denaturation step at $94^{\circ} \mathrm{C}$, (2) $10 \mathrm{sec}$ annealing at $62^{\circ} \mathrm{C}$, and (3) $30 \mathrm{sec}$ extension at $72^{\circ} \mathrm{C}$. A Perkin-Elmer model gene Amp PCR system 2400 was used. To obtain a semiquantitative analysis, primers specific for the glyceraldehyde-3-phosphate dehydrogenase (GAPDH) housekeeping gene (forward: GACCACAGTCCATGACATCACT; reverse: TCCACCACCCTGTTGCTGTAG; 20 pmol each) were added to the PCR reactions; the GAPDH cDNA was used as internal standard to normalize the concentration of the different cDNA samples. Because the GAPDH mRNA was more abundant than the sialidase mRNA, the sialidase cDNA was allowed to undergo six initial cycles of amplification before addition of GAPDH primers. Under those PCR conditions, none of the cDNAs was amplified to saturation.

A densitometric analysis of the band intensities was performed using the NIH Image software.

Cell culture. Primary hippocampal neurons derived from rat embryos were cultured following the protocol of Goslin and Banker (1991) and de Hoop et al. (1997). In brief, the hippocampi of E18 rats were dissected, trypsinized, and physically dissociated. The cells were then washed in HBSS, and 100,000 cells (25,000 for neurite length measurements) were plated onto poly-L-lysine (PLL) or laminin-treated glass coverslips in 6 $\mathrm{cm}$ Petri dishes containing minimal essential medium (MEM) and $10 \%$ heat-inactivated horse serum. The cells were kept in $5 \% \mathrm{CO}_{2}$ at $36.5^{\circ} \mathrm{C}$. After $7 \mathrm{hr}$ the coverslips were transferred to a $6 \mathrm{~cm}$ dish containing astrocytes in MEM and N2 supplements containing $0.5 \mathrm{~mm} 2$,3, dehydro2 , deoxy- $N$-acetylneuraminic acid (NeuAc2en) in the case of PMGS inhibitor-treated cultures, or $10 \mu \mathrm{g} / \mathrm{ml}$ cholera toxin-subunit B in GM1 binding-blocking experiments.

For biochemical purposes cells were plated onto $3 \mathrm{~cm}$ tissue culture dishes containing MEM-N2 medium and incubated the same way as before.

COS cells were plated in $3 \mathrm{~cm}$ Petri dishes (10,000 cells per dish) containing DMEM supplemented with $10 \%$ heat-inactivated fetal calf serum (FCS).

Axon transection. One-day-old neurons either transfected or nontransfected with the PMGS cDNA (see below) were plated on PLL-treated Cellocate coverslips (Eppendorf, Hamburg, Germany). The coverslips were then transferred into a tissue culture dish filled with medium equilibrated to air concentration of gases onto a microscope stage equipped with a micromanipulator (Eppendorf). Cells were localized on the coverslip grid using a $32 \times$ long-distance working lens. The longest neurite was then cut by pulling, orthogonally across the neurite, a microinjection glass needle over the glass surface. The distance between the cell body and the cut site was similar to the second longest neurite. Cells were photographed before and shortly after lesioning. The coverslip was then placed in the original medium and returned to the incubator. After $24 \mathrm{hr}$, cells were fixed and labeled with anti-hemagglutinin (HA) antibody (see below). Lesioned cells were then relocalized and newly photographed.

Immunocytochemistry. For the detection of tubulin-, MAP-2-, tau-, and HA-tagged PMGS, cultured neurons were fixed in $4 \%$ paraformaldehyde for $15 \mathrm{~min}$ at room temperature, aldehyde groups were quenched in $50 \mathrm{~mm}$ ammonium chloride for $5 \mathrm{~min}$, and the cells were then extracted with $0.1 \%$ Triton X-100 for 3 min. The neurons were blocked in blocking solution for $1 \mathrm{hr}$ at room temperature and then incubated with rabbit anti-HA (Santa Cruz Biotechnology, Santa Cruz, CA), polyclonal antibody 514 anti-MAP2 (a gift from C. Sanchez, Centro de Biologia Molecular, Madrid), mAb anti-tau (Tau-1, Roche Diagnostics, Mannheim, Germany), and mAb anti-tubulin (Amersham Pharmacia Biotech). Incubations were performed for $1 \mathrm{hr}$ at room temperature. After extensive washing with PBS, cells were incubated with species-specific secondary antibodies.

Western blotting. Samples of plasma membranes of neuronal or COS cells were dissolved in Laemmli's sample buffer $(5 \times)$ and loaded onto
$12 \%$ polyacrylamide PAGE-SDS gels ( $30 \mu \mathrm{g}$ of protein per line). The proteins were transferred to nitrocellulose filters and blocked for $2 \mathrm{hr}$ with $5 \%$ defatted milk, $0.1 \%$ Tween 20 in PBS (PBST) at room temperature. The filters were then incubated overnight at $4^{\circ} \mathrm{C}$ with polyclonal antibodies raised against PMGS or with polyclonal anti-HA antibodies (Santa Cruz Biotechnology). After they were washed thoroughly with PBST, the filters were incubated with horseradish peroxidase-labeled anti-rabbit antibodies for $1 \mathrm{hr}$ at room temperature, washed, and developed by the ECL system (Amersham Pharmacia Biotech).

Neurite length measurements. Control or NeuAc2en-treated $(0.5 \mathrm{~mm})$ neuron cultures were labeled with $\mathrm{mAb}$ anti-tubulin as described before, and 20 random fields per experiment were photographed using a Zeiss Axiovert 135 microscope equipped with an acrostigmat $20 \times$ objective.

The neurite length (80-100 cells per experiment) was measured from the cell body to the growth cone using NIH Image 1.58 software. For axon transection experiments, the axonal regrowth was measured from the cut site to the new growth cone. In the case of growth of a different neurite, the measurement was from the tip of the growing neurite before the lesion to the growth cone $24 \mathrm{hr}$ after. The grid of the Cellocate coverslips was used as reference.

Expression of HA-tagged murine plasma membrane ganglioside sialidase in COS-1 cells and hippocampal neurons. A sialidase expression plasmid coding for the murine PMGS with an HA-tag at the C terminus was constructed as follows. Two primers, (1) 5'-AAGCTTGCCACCATGGAGGAAGTCCCACCC, introducing a HindIII site and a Kozac (Kozac, 1989) sequence, and (2) 5'-GCGGCCGCCCGTCGCTACTAGGGCTG, introducing a NotI site, were used to amplify the sequence encoding the entire open reading frame from template cDNA synthesized from mouse brain poly $\left(\mathrm{A}^{+}\right)$RNA (Hasegawa et al., 2000). PCR products were cloned into a pCRII-TOPO vector (Invitrogen, San Diego, CA) following manufacturer's instructions. The sialidase was then subcloned into PMH expression vector (Roche Diagnostics, Mannheim, Germany) using HindIII and NotI restriction sites. The construct was verified by sequencing.

To verify the activity of the sialidase encoded by this construct, $3 \times 10^{6}$ COS-1 cells per milliliter were transfected by electroporation with the empty expression vector or with the construct $(20 \mu \mathrm{g}$ of DNA/300 $\mu \mathrm{l}$ of cell suspension) using a Bio-Rad (Bio-Rad, Hercules, CA) Genepulser operated at $250 \mathrm{~V}$ and $500 \mu \mathrm{F}$. After $3 \mathrm{~d}$ in culture as described above, the cells were washed with PBS, scraped, pelleted, lysed by ultrasonication in 9 vol of cold PBS, and centrifuged at $1000 \times g$ for $10 \mathrm{~min}$ at $4^{\circ} \mathrm{C}$. The supernatant was further centrifuged at $100,000 \times g$ for $1 \mathrm{hr}$ at $4^{\circ} \mathrm{C}$. The pellet was used in the sialidase assay as the membrane fraction. Rat hippocampal neurons were transfected in the same way as COS cells, but the capacitance was set to $250 \mu \mathrm{F}$.

Sialidase assay: ganglioside analysis and sialic acid quantification. The assay mixture contained $50 \mathrm{nmol}$ of ganglioside-bound sialic acid (bovine mixed gangliosides; Matreya Inc.), $0.2 \mathrm{mg}$ of BSA, $15 \mu \mathrm{mol}$ of sodium acetate, $\mathrm{pH} 4.6,0.2 \mathrm{mg}$ of Triton X-100, and membrane samples in $0.2 \mathrm{ml}$ (plasma membranes from $3 \times 10^{6} \mathrm{COS}$ cells or $5 \times 10^{6}$ primary neurons). After $1 \mathrm{hr}$ incubation at $37^{\circ} \mathrm{C}$, the reaction was taken up to $2 \mathrm{ml}$ with water and loaded on a C18 reversed-phase column (Merck, Darmstadt, Germany) as described by Reuter and Schauer (1994). Released sialic acid was recovered in the nonretained fraction, whereas gangliosides were recovered by elution with methanol. Aqueous nonretained fraction was lyophilized and methanol fraction was vacuum dried.

Eluted gangliosides were resuspended by sonication in $30 \mu \mathrm{l}$ of methanol and chromatographed on a high-performance thin-layer chromatographic plate (Merck, Darmstadt, Germany) in chloroform/methanol/ $0.2 \% \mathrm{CaCl}_{2}(55: 45: 10 \mathrm{v} / \mathrm{v} / \mathrm{v})$. Ganglioside bands were visualized with resorcinol-HCl reagent as described by Schnaar and Needham (1994). Released sialic acid was measured by the method of Warren (1959), as modified by Chaplin (1986), using $N$-acetylneuraminic acid as standard.

\section{RESULTS \\ PMGS mRNA, protein, and activity are high at early developmental stages}

Taking into account that addition of GM1 to cultured neuronal cells facilitates axonal growth (see introductory remarks), we predicted that the levels and activity of the enzyme responsible for its plasma membrane synthesis should be higher at developmental stages when axonal growth is the maximum. To test this hypothesis we measured PMGS mRNA, protein, and activity in 
hippocampal neurons in situ and in vitro. Template cDNA from embryonic (E15) and adult (2-month-old) mouse hippocampi was PCR amplified with PMGS gene-specific primers (see Materials and Methods). The abundance of GAPDH mRNA in the two samples was used as internal standard to normalize the concentration of the cDNA samples. The expression level of PMGS mRNA was significantly higher at earlier developmental stages (Fig. $1 A$ ). The bar graph in Figure $1 A$ shows the quantification of the relative abundance of PMGS mRNA in embryonic versus adult hippocampi in four independent experiments. Although a certain variability in the values from different experiments was observed, PMGS transcripts were reproducibly more enriched at the embryonic hippocampal stage $(10.5 \times$ on average) (Fig. $1 \mathrm{~A}$, black bar). The result of one representative experiment is shown in the bottom panel of Figure $1 \mathrm{~A}$.

The levels of PMGS protein were investigated in embryonic hippocampal neurons grown in culture conditions, a well suited experimental system for neuronal differentiation studies (for review, see Craig and Banker, 1994; Bradke and Dotti, 2000). Western blotting of the membrane fraction of neurons grown for 1 and $3 \mathrm{~d}$ in either laminin or PLL was performed using a polyclonal anti-PMGS antibody (Fig. 1B). PMGS levels in laminin-grown cells were higher than from cells grown in PLL. Moreover, the levels were significantly higher in cells grown for $3 \mathrm{~d}$ on PLL than for those grown for $1 \mathrm{~d}$. The difference between neurons seeded on laminin or PLL can be explained by the fact that laminin is a naturally occurring extracellular matrix protein present in the hippocampus and that these cells grow axons much faster than the axons of the same neurons grown on PLL (Manthorpe et al., 1983; Lein et al., 1992; Esch et al., 1999).

The hydrolyzing capacity of PMGS at different developmental stages was also tested in membranes of hippocampal neurons grown for different times and on different substrata. Quantitative analysis of the sialic acid hydrolyzed by membranes of neurons cultured on PLL or laminin is shown in Figure $1 C$. The membranes from neurons grown on laminin for 1 and $3 \mathrm{~d}$ showed between 2.5- and 3-fold higher activity than in the case of cells grown for $3 \mathrm{~d}$ on PLL (Fig. 1 C, PMGS activity). Enzymatic levels were undetectable in neurons grown for $1 \mathrm{~d}$ on PLL. Considering that by day 1 hippocampal neurons grown on PLL are just sprouting neurites, and only by day 3 are a large number of them actively elongating axons (Dotti et al., 1988), the patterns of expression just described show the existence of a temporal correlation between PMGS high levels and neuritogenesis. Whether the activity of the enzyme plays a direct role in neuritogenesis was tested next in gain and loss of function experiments.

\section{Inhibition of neuronal PMGS activity diminishes neurite growth}

PMGS hydrolyzes sialic acid from plasma membrane oligosialogangliosides, releasing $\alpha 2-3$ - and $\alpha 2-8$-bound sialic acids to thus produce plasma membrane GM1 (Saito et al., 1995; Kopitz et al., 1996; Hata et al., 1998, Miyagi et al., 1999). To inhibit the activity of PMGS, cultured hippocampal neurons grown on laminin or PLL were treated with the specific inhibitor NeuAc2en. Studies in different cell types have already shown that the activity of the enzyme is blocked with this inhibitor (Kopitz et al., 1996, 1997). NeuAc2en was added to the laminin-seeded neurons shortly after attachment ( $6 \mathrm{hr}$ in vitro), and the effect on axonal and dendritic length and number was measured $18 \mathrm{hr}$ later. Untreated laminingrown neurons showed long axons (Fig. $2 C$, arrowheads). Addition of NeuAc2en to sister neurons drastically affected this phe-
A
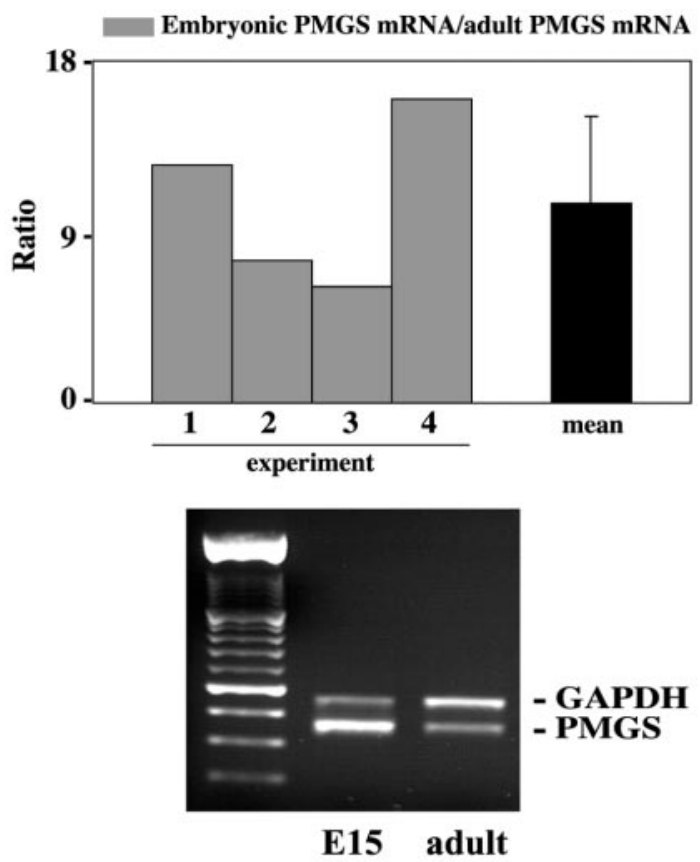

B

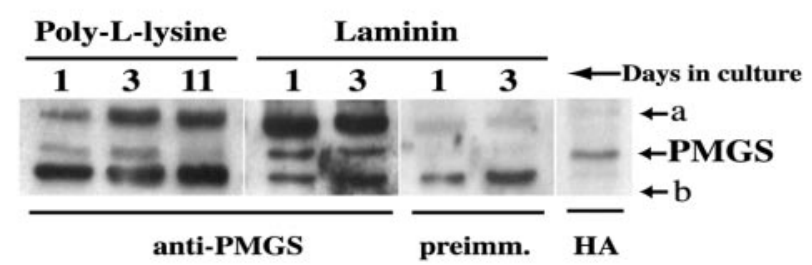

$\mathbf{C}$

Poly-L-lysine Laminin

\begin{tabular}{|c|c|c|c|c|c|}
\hline 1 & 3 & 11 & 1 & 3 & $\leftarrow$ Days in culture \\
\hline .d. & 10 & n.d. & 28 & 25 & MG \\
\hline
\end{tabular}

(nmol sialic acid/hour $\mathbf{x}$ mg total prot.)

Figure 1. PMGS is highly expressed during early stages of neuronal differentiation. $A$, Quantification of the relative enrichment of PMGS mRNA in four independent RT-PCR experiments. Semiquantitative RTPCR analysis shows that PMGS is significantly more expressed in embryonic than in adult hippocampi. Each gray bar represents the ratio of PMGS mRNA concentration in embryonic hippocampi (E15) over that found in adult hippocampi (postnatal day 60) for a given experiment, as measured by densitometric analysis of the band intensities. The mean is indicated by the black bar. Error bar corresponds to the SD. The bottom panel shows the result of a representative experiment. The expression level of the housekeeping gene GAPDH was used to normalize the mRNA concentration. $B$, Western blot using polyclonal anti-PMGS antibody of membrane extracts from hippocampal neurons grown on PLL or on laminin-coated dishes. Neurons grown on laminin express much higher levels of PMGS than those grown on PLL. In this case the higher expression occurs after $3 \mathrm{~d}$ in culture. A second band (labeled $a$ ) of $\sim 5$ $\mathrm{kDa}$ higher molecular weight could represent a post-translational modification. The two lanes labeled preimm. show the reprobing with preimmune serum of the blot for laminin-grown cells. This demonstrates that the bottom band (labeled $b$ ) is nonspecific. In the right lane $(H A)$, we localized the PMGS band by probing membranes from HA-PMGSoverexpressing neurons with anti-HA antibody. $C$, Quantification of PMGS activity in membranes from PLL- and laminin-grown cells. Sialic acid release from a mixture of oligosialogangliosides of such confirmed the high activity of the enzyme in laminin-grown neurons.

notype, and most neurons presented only short neurites (Fig. 2D, arrowheads). Quantification of the effect of NeuAc2en was performed measuring neuritic length of treated and control cultures and splitting the measurements into three categories: short neu- 

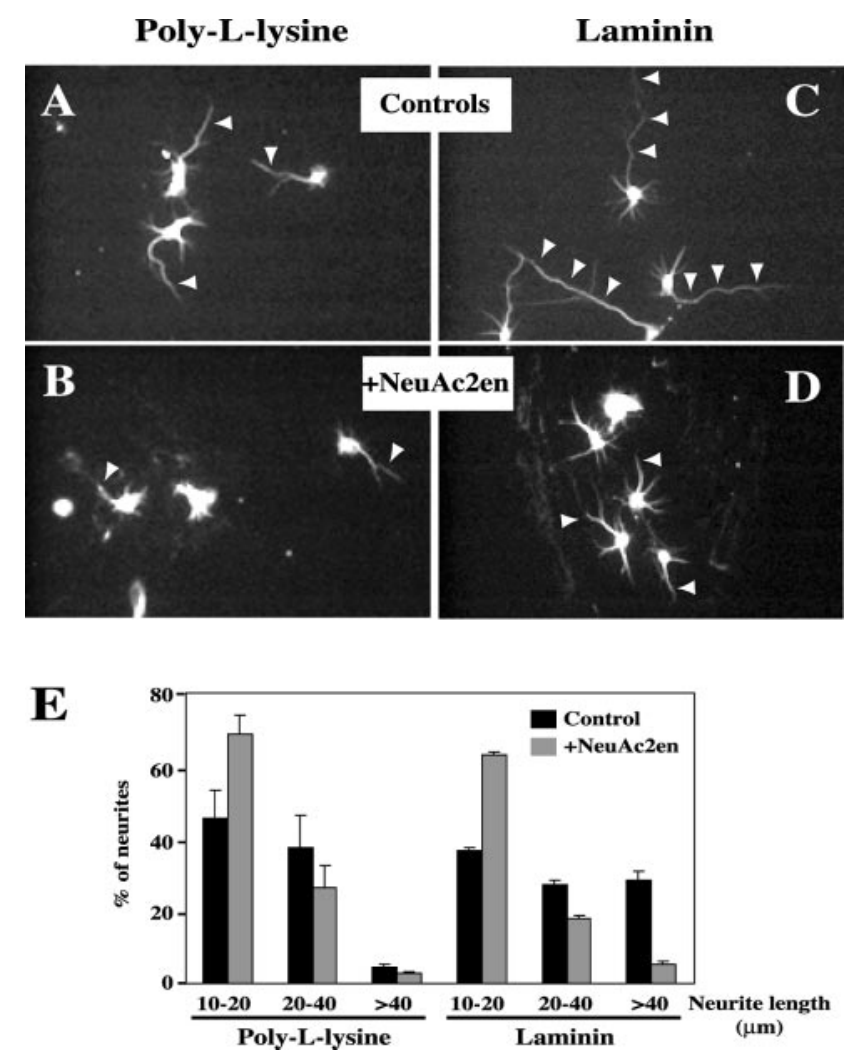

Figure 2. Inhibition of PMGS activity retards axonal growth. $A-D$, Morphological appearance of hippocampal neurons grown on PLL $(A, B)$ or on laminin $(C, D)$ in the presence $(B, D)$ or absence $(A, C)$ of $0.5 \mathrm{~mm}$ NeuAc2en. The inhibitor was added $6 \mathrm{hr}$ after plating the cells and incubated for $18 \mathrm{hr}$. All the cells were stained with monoclonal antitubulin antibody to visualize the processes. Note the poor differentiation of cells treated with the inhibitor. $E$, Quantification of neuritic length of the experiment shown above. Neuritic length was measured as indicated in Materials and Methods. The results were divided in three categories: 10- to $20-\mu \mathrm{m}$-long neurites, 20 - to $40-\mu \mathrm{m}$-long neurites, and neurites $>40$ $\mu \mathrm{m}$. Black bars and gray bars represent the percentage of neurites in each category for control and treated cultures, respectively. The results correspond to the average of three experiments. Error bars correspond to the SD.

rites $(10-20 \mu \mathrm{m})$, neurites of intermediate length $(20-40 \mu \mathrm{m})$, and clearly identifiable axons $(>40 \mu \mathrm{m})$. NeuAc2en treatment reduced the number of axons from $31 \%$ (untreated) to $7 \%$, whereas the number of short neurites was enhanced from $41 \%$ (control) to $72 \%$ (Fig. $2 E$, three bar sets on the right). Although to a lesser extent, a similar inhibitory effect was observed on PLLcultured neurons (Fig. $2 A, B, E$, three bar sets on the left). This latter effect was not surprising because axonal growth is much slower on PLL than on laminin (Manthorpe et al., 1983). Similar results were obtained blocking GM1 with the subunit B of cholera toxin (see Fig. 5A,B).

To confirm that the observed effect of NeuAc2en was caused by a true reduction of surface GM1, we treated hippocampal neurons in culture and analyzed ChTx-B binding by immunofluorescence. A treatment identical to that performed for the functional analysis (see above) resulted in an $8 \times$ loss of FITC-cholera toxin-subunit B binding (data not shown).

\section{Increase of PMGS enzymatic activity accelerates axonal growth}

Because inhibition of PMGS activity blocks GM1 synthesis and axonal elongation (Fig. 2), we hypothesized that an increase in
PMGS activity would produce neurons with longer neurites. Before testing this hypothesis in neurons, we controlled in COS-1 cells, which are more manageable for biochemical analysis, if PMGS overexpression resulted in a true, higher enzymatic activity. Cell were transfected with the PMGS plasmid, and the distribution and activity of the newly made protein were analyzed by immunofluorescence microscopy, Western blotting, and thinlayer chromatography. Two days after transfection, overexpressing cells showed intracellular and membrane labeling with the anti-HA antibody (Fig. $3 A$ ). The presence of the protein in the membrane fraction was also demonstrated by Western blotting (Fig. 3A). Enzymatic activity was analyzed using a mix of oligosialogangliosides as substratum (see Materials and Methods). The plasma membranes of transfected cells released $69.3 \mu \mathrm{mol}$ of sialic acid per hour per milligram of protein (Fig. 3C, PMGS). The enzymatic activity of plasma membranes from vector-alone transfected cells was undetectable (Fig. $3 C$, vector). In agreement with this quantification, the thin-layer chromatography in Figure $3 B$ shows qualitatively how the membranes from transfected cells $(P M G S)$ efficiently hydrolyzed the oligosialogangliosides in the original mixture producing GM1. Contrarily, the membranes from cells transfected with the expression vector alone or from nontransfected cells did not show such activity (Fig. 3B, vector and control, respectively).

Once we demonstrated that the enzyme encoded by our HAPMGS construct was properly located and fully functional, we tested the effect of PMGS overexpression in the differentiation of hippocampal neurons in culture. Neurons grown on either laminin or PLL were transfected with the above construct, and neuritic number and length were measured $24 \mathrm{hr}$ later. In the control situation (empty expression vector) using PLL as substratum, most neurons were in stage 1 (round, no processes) or stage 2 (several short neurites), and very few were in the polarized stage 3 (one long and several short neurites) (Dotti et al., 1988). Quite dramatically, under the same condition of culture, PMGS overexpression in neurons (Fig. $4 A-D$, arrows) from a parallel culture resulted in the appearance of numerous neurons with one or two very long axon-like neurites $(>40 \mu \mathrm{m})$ (Fig. $4 A-D$, arrowheads). Quantitation of neuritic number and length revealed that overexpression increased the number of processes $>40 \mu \mathrm{m}$ from $7 \%$ (untransfected) to $37 \%$. Consistently, the number of neurites of intermediate length was strongly decreased (26\% for overexpressing cells and $52 \%$ for controls) (Fig. 4E, central bar set). This effect could be reverted by blocking GM1 with cholera toxinsubunit B (Fig. 5C). Together with the previous data on PMGS inhibition, these last results strongly suggest the involvement of PMGS in axonal growth.

\section{Overexpression of PMGS promotes early axonal and dendritic cytoskeleton segregation}

To determine whether PMGS-mediated acceleration in growth rate also accelerates overall neuronal maturation, we analyzed the distribution of the microtubule-associated proteins tau and MAP2 in control and PMGS-overexpressing neurons. These proteins become segregated to axons and dendrites, respectively, not earlier than 4-5 d in culture (Caceres et al., 1984, 1986). Figure $6 A$ shows a control neuron grown in culture for $72 \mathrm{hr}$, immunostained for tau and MAP2. Consistent with previous work at this time in culture, both proteins were uniformly distributed to the axon and dendrites. Conversely, PMGS- overexpressing neurons presented a clear axonal polarization of tau (Fig. 6B, arrowheads) and dendritic polarization of MAP2 (Fig. 6C, arrowheads) as 
$\mathbf{A}$
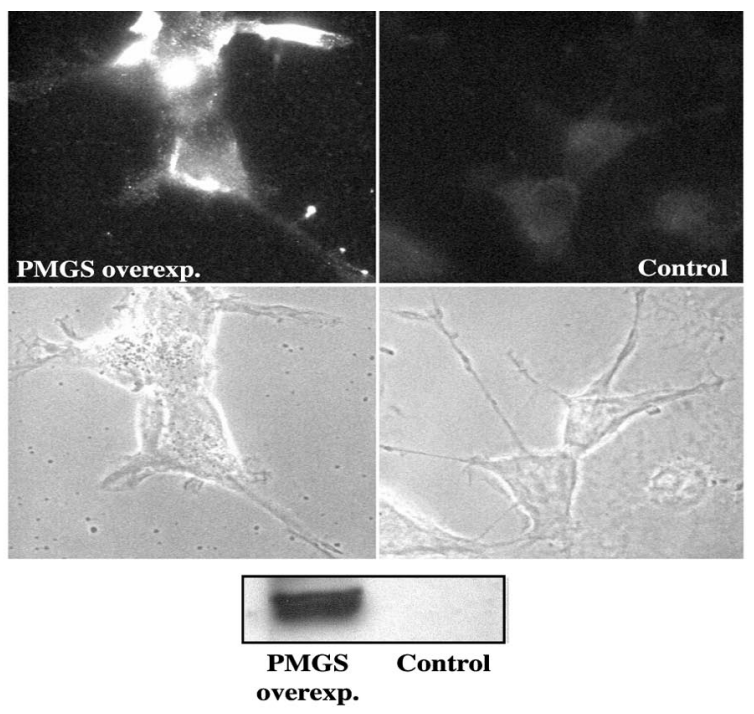

B

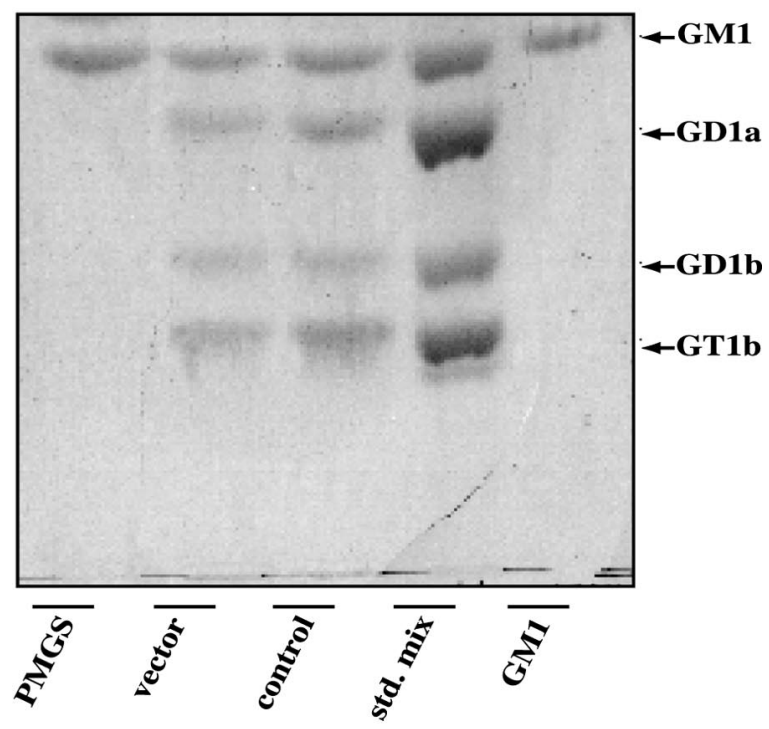

C

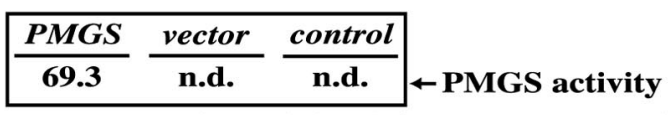

(nmol sialic acid/hour $x$ mg total prot.)

Figure 3. Overexpressed HA-PMGS is properly localized and fully functional. $A$, COS-1 cells were transfected with HA-PMGS cDNA (see Materials and Methods for details) and grown for $48 \mathrm{hr}$. The immunofluorescence with anti-HA antibodies shows a strong expression of HAPMGS on the plasma membrane (A, left panel). A Western blot with the same antibody confirmed a high level of expression for plasma membranes from transfected cells (inset, PMGS overexp. lane), whereas for membranes from control cells the expression is undetectable (inset, Control lane). $B, C$, Plasma membranes from PMGS-transfected or vectortransfected COS-1 cells were incubated with a mixture of standard gangliosides in the presence of Triton X-100. The resulting gangliosides and the released sialic acid were separated by reversed-phase chromatography. Gangliosides were qualitatively analyzed by thin-layer chromatography with resorcinol staining $(B)$, and sialic acid was quantitated using a thyobarbituric acid assay $(C)$. Membranes from PMGS-transfected cells hydrolyzed all the polysialilated gangliosides forming GM1 and releasing $69.3 \mathrm{nmol}$ of sialic acid per hour per milligram of protein (PMGS lane). In the case of membranes from vector-transfected cells (vector lane), as in a control without membranes (control lane), polysialilated gangliosides remained undigested, and released sialic acid could not be detected (n.d., nondetectable).
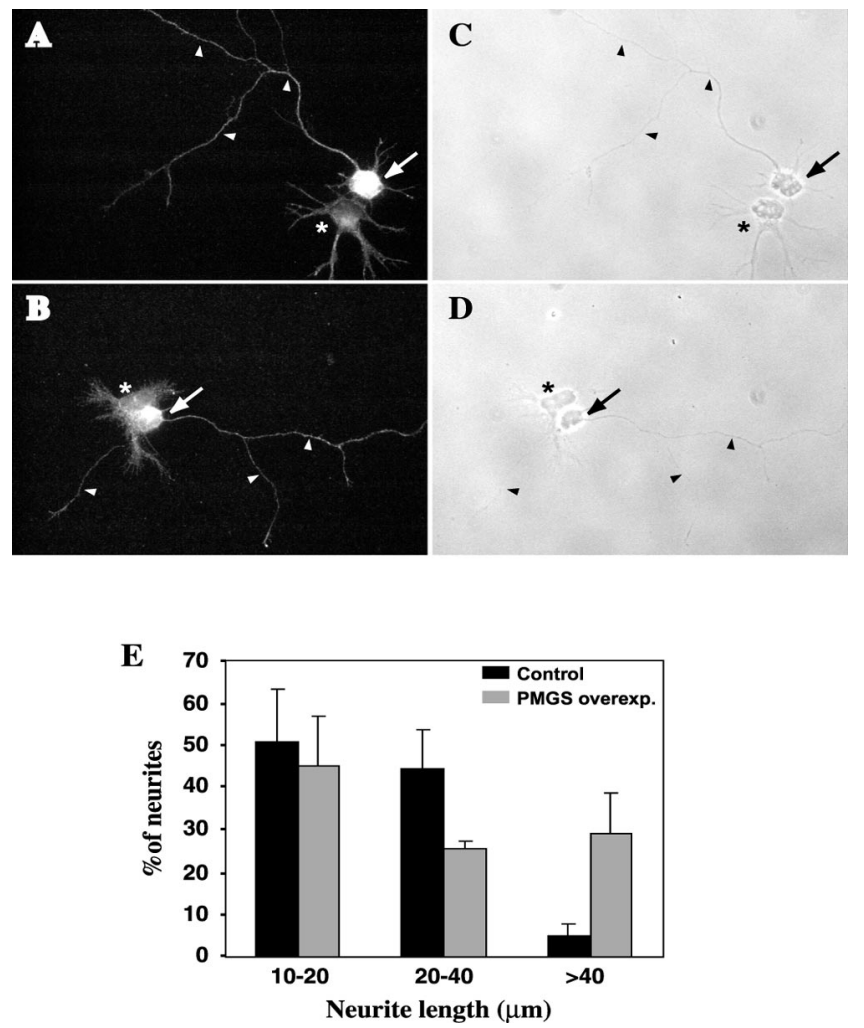

Figure 4. PMGS overexpression accelerates axonal growth in cultured hippocampal neurons. $A-D$, Hippocampal neurons were transfected with HA-PMGS cDNA and plated on PLL-coated coverslips. After $24 \mathrm{hr}$ the cells were fixed and immunolabeled with anti-HA antibodies. To better illustrate the effect of overexpression, pairs of neurons overexpressing HA-PMGS (arrows) and nontransfected (asterisks) neurons are shown. The overexpressing neurons showed long and branched axons $(A, B$, arrowheads), whereas non-overexpressing cells $(A, B$, asterisks) presented only short neurites. $E$, Quantitation of neurite length for control ( gray bars) and PMGS-overexpressing neurons (black bars) after $24 \mathrm{hr}$ in culture. PMGS-overexpressing neurons showed $38 \%$ of axons $>40 \mu \mathrm{m}$, whereas this group represents only $7 \%$ for control cells. The intermediate group of neurites, on the contrary, was significantly reduced from $51 \%$ in controls to $26 \%$ in transfected cells. The quantification was made on a total of 100 overexpressing cells and 100 control cells from three independent experiments. We considered a cell as "overexpressing" when the anti-HA fluorescence intensity of the cell body was $>2.5$ times the background level measured on nontransfected cultures. Error bars correspond to the SD.

early as $36 \mathrm{hr}$ after plating. These results suggest that the acceleration of axonal growth driven by the increased expression of PMGS and GM1 speed up neuronal maturation, at least regarding polarization of cytoskeletal proteins.

\section{PMGS overexpression increases the regeneration capacity of the initial axon}

Given that PMGS overexpression enhanced axonal growth and maturation, it became essential to investigate whether the response to axotomy was modified as well. To address this question we cut the axons of stage 3 PMGS-overexpressing hippocampal neurons and analyzed their capacity to regenerate. Axons were sectioned in such a way that the proximal stump was left with a length similar to or shorter than that of the other neurites (Fig. $7 A, B$, white lines). The rationale behind such procedures was to return the neuron to a stage in which all processes would have similar chances to become the new axon (Goslin and Banker, 1991). Consistent with that concept, lesioned control neurons 


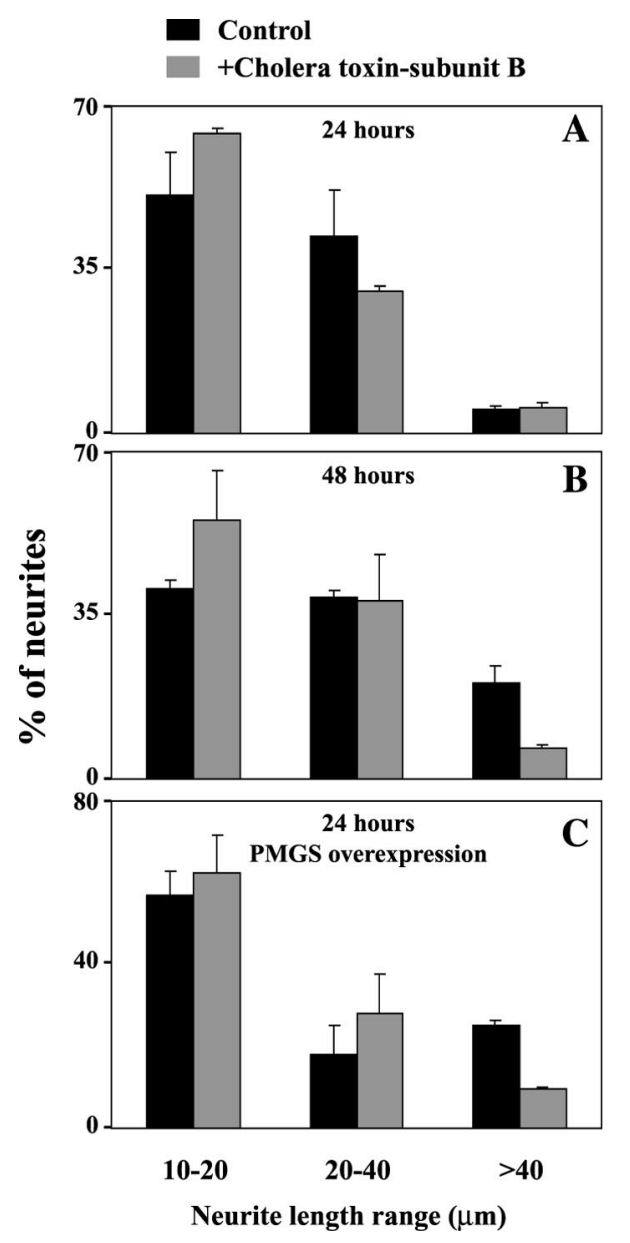

Figure 5. Inhibition of surface GM1 binding with cholera toxin-subunit $\mathrm{B}$ retards early axonal growth and reverts the effect of PMGS overexpression. Control ( $A, B$, black bars) or PMGS-overexpressing hippocampal neurons $(C$, black bars) grown on PLL were treated with $10 \mu \mathrm{g} / \mathrm{ml}$ cholera toxin-subunit $\mathrm{B}(A-C$, gray bars). The reagent was added $6 \mathrm{hr}$ after plating and maintained for 18 or $42 \mathrm{hr}$. After incubation the cells were fixed at 24 $(A, C)$ or $48 \mathrm{hr}(B)$ in paraformaldehyde and photographed. Neuritic length was measured as indicated in Materials and Methods. The results from two independent experiments were divided into three categories: 10 - to $20-\mu \mathrm{m}$-long neurites, 20 - to $40-\mu \mathrm{m}$-long neurites, and neurites $>40$ $\mu \mathrm{m}$. Error bars correspond to the SD.

regrew a new axon from a neurite different from that sectioned (Fig. 7A, black arrowhead, white arrowheads). On the contrary, a similar experiment in PMGS-overexpressing cells resulted in the growth of a new axon from the sectioned axonal stump (Fig. $7 B$, white and black arrowheads). Seventy percent of control lesioned neurons grew a different axon after axotomy. Contrarily, in $71 \%$ of PMGS-overexpressing neurons, the sectioned axon regrew as such (Fig. 7C). To demonstrate further the specificity of this effect, PMGS-overexpressing cells were incubated in the presence of the PMGS inhibitor NeuAc2en and then axotomized. Under these conditions $60 \%$ of PMGS-overexpressing neurons grew a different axon (Fig. 7C, right bar set).

\section{DISCUSSION}

In this work we show that PMGS mRNA and protein levels are significantly higher in hippocampal neurons at early stages of development than at late stages, both in situ and in culture conditions. By virtue of the role of PMGS in the synthesis of plasma membrane GM1 (Miyagi et al., 1999; Wada et al., 1999;
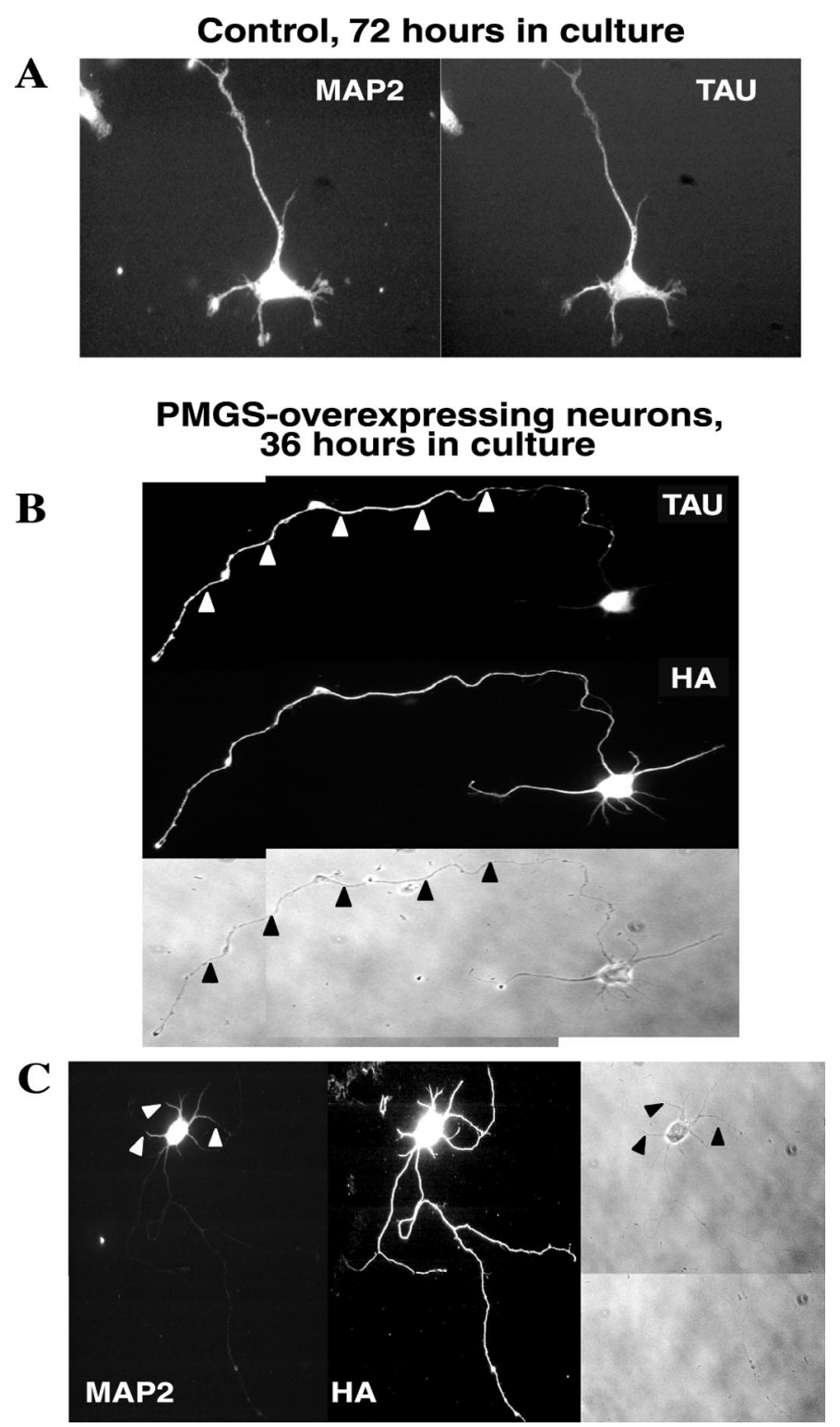

Figure 6. PMGS overexpression produces an early polarization of the axonal marker tau and the dendritic marker MAP2. A, Hippocampal neurons were plated on PLL-coated coverslips, fixed after $72 \mathrm{hr}$ in culture, and double immunostained for tau and MAP2. At this moment the expression of both markers appeared evenly distributed along the whole cell. $B, C$, Hippocampal neurons transfected with HA-PMGS cDNA were fixed after $36 \mathrm{hr}$ in culture and double immunostained for tau and HA $(B)$ or HA and MAP2 $(C)$. In these cells tau was clearly polarized to the axon (B, arrowheads), and MAP2 was clearly polarized to the somatodendritic domain $(C$, arrowheads).

Hasegawa et al., 2000), these first results support the notion that plasma membrane GM1 plays an important role in the early stages of neuronal differentiation. In what concerns the well characterized hippocampal neurons in primary culture, one of such differentiation roles seems to be the regulation of axonal growth. In support of this, we here show that addition of the specific inhibitor of PMGS activity, NeuAc2en, reduced GM1 plasma membrane levels and arrested axonal growth. Similar axonal growth retardation was observed blocking GM1 directly with cholera toxin-subunit B. Consistently, overexpression of the murine PMGS cDNA enhanced GM1 synthesis and facilitated 


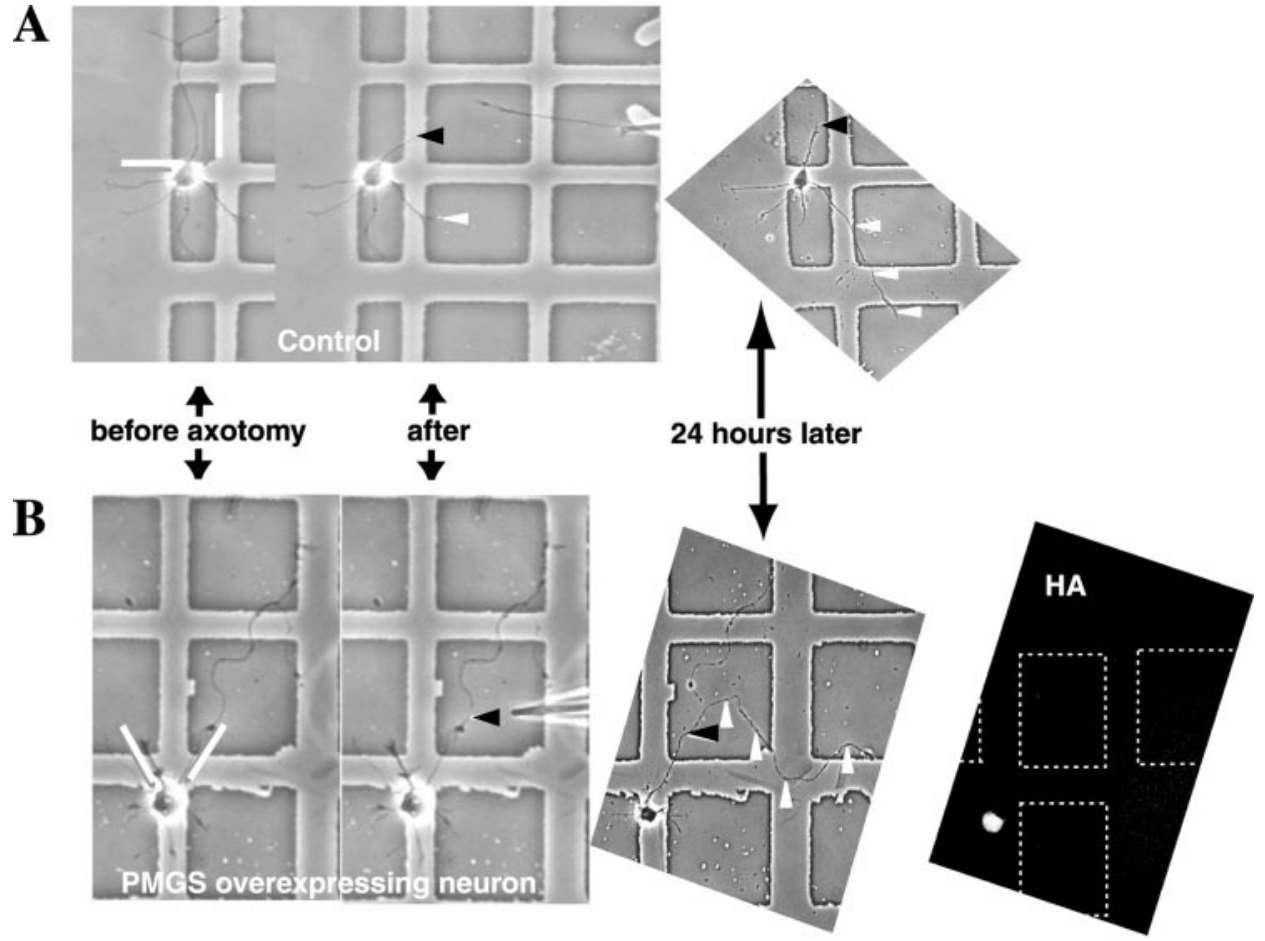

C

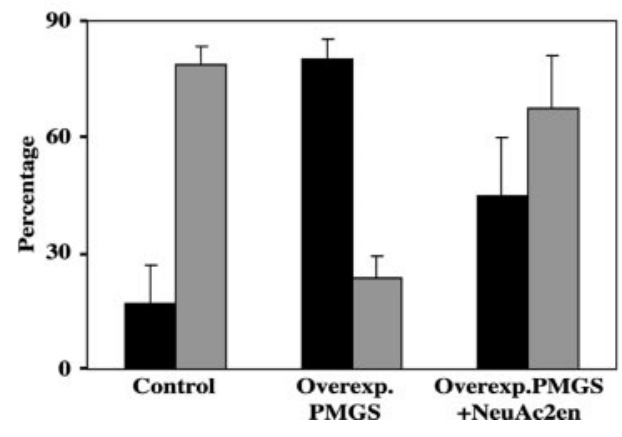

Figure 7. PMGS-overexpressing neurons regenerate an axon from the same process originally sectioned, even after proximal axotomy. $A$, Hippocampal neurons were plated on PLL-coated Cellocate coverslips and grown for $24 \mathrm{hr}$. At that time the axons were sectioned at a distance from the cell body similar to the length of the longest dendrite ( $A$, before axotomy, white lines). Twenty-four hours after the sectioning the cells were fixed and relocalized using the Cellocate grid as reference. The sectioned axons appeared stacked at the point where they were cut $(A, 24$ hours later, and black arrowhead), and one of the dendrites grew as the new axon $(A, 24$ hours later, and white arrowheads). $B$, The axons of HA-PMGS-transfected neurons were sectioned and allowed to recover as for cells in $A$; then the cells were fixed and immunolabeled with anti-HA antibodies. Twenty-four hours after axotomy, the same axons grew again from the point of the cut ( $B, 24$ hours later, arrowheads), and the dendrites remained unaltered. $C$, Axonal regeneration in PMGS-overexpressing cells can be partially reversed by the PMGS inhibitor NeuAc2en. From the experiments described in $A$ and $B$, we quantitated the number of cells from which a new axon grew from a different neurite ( gray bars) or from the same process (black bars). Seventy percent of the control cells grew a new axon from a different neurite; conversely, $71 \%$ of the PMGSoverexpressing neurons (Overexp. PMGS) regrew from the same process (means of three experiments). When overexpressing neurons were treated with $0.5 \mathrm{~mm}$ NeuAc2en (Overexp.PMGS + NeuAc2en), the effect was partially reversed, and $60 \%$ of the cells grew a different neurite after axotomy (mean of two experiments). Error bars correspond to SDs. axonal growth. Additionally, we show that PMGS overexpression causes an early polarization of cytoskeletal components such as MAP2 or tau. The simplest explanation for such early polarization is the overall acceleration of neuronal maturation triggered by high expression of PMGS. However, without analysis of synaptic activity, such a conclusion at this moment is a simple working hypothesis. Although our NeuAc2en data do not permit us to exclude the involvement of GM1 in dendritic growth, the PMGS overexpression experiment suggests that, at least at these early stages, PMGS-GM1 is especially important for axon growth. Indeed, we observed that all overexpressing neurons had long axons, but the dendrites were no longer than those of non-overexpressing neurons. Because our own overexpression experiments with tagged axonal growth-unrelated membrane proteins (i.e., amyloid precursor protein, synaptophysin) (Kaether et al., 2000) did not affect axonal elongation, the results presented here point to the specific "axonogenic" role of PMGS.

We also observed that PMGS is expressed at much higher levels in neurons grown on laminin than in those grown on PLL and that the inhibitory effect of NeuAc2en is more evident in neurons grown on laminin than on those grown on PLL. Because the hippocampal region is rich in laminin (Nakagami et al., 2000) and hippocampal neurons express integrin receptors (Pinkstaff et al., 1999), the expression and functional data here reported raise the possibility that PMGS may modulate axonal growth through an increase in the efficiency of integrin-laminin interaction (McKerracher et al., 1996). Although we present no experimental evidence in favor of this argument, except for the correlation just reported, such modulation may be similar to that between GM1 and the extracellular matrix protein galectin-1 in the olfactory tract, an event essential for the enhancement of axonal growth and proper directionality toward the olfactory bulb (Puche et al., 1996; Kopitz et al., 1998).

The mechanisms by which the increase in surface PMGS levels and activity modulate growth remain to be investigated. It is reasonable to think that an axonal growth determinant acts on surface GM1 (see introductory remarks), resulting in a signaling event that produces an increase in PMGS synthesis and insertion in the axonal membrane. This local increase in PMGS would then be followed by an increase in GM1 interaction with the ligand, thus stimulating changes in the dynamics of membrane fusionretrieval and actin and microtubule cytoskeleton that are needed to support further growth. Consistent with the above scenario are the following observations. (1) PMGS expression levels increase in neuroblastoma cells exposed to differentiation agents (Hasegawa et al., 2000) and in hippocampal neurons grown under 
conditions in which axonal differentiation is favored (this work). (2) Alteration of GM1 concentration in detergent-insoluble glycosphingolipid-enriched domains (rafts) or its interaction with antibodies (Ravichra and Joshi, 1999) affects the phosphorylation state of some of the signaling proteins associated with these domains, leading to changes in transmembrane signaling. (3) Neuron-like cells treated with exogenous GM1 show enhanced neuritogenesis accompanied by a disruption of the actin network and an increased association of the MAP2 with actin filaments (Wang et al., 1998). It is then conceivable that the localized increase in PMGS-GM1 leading to accelerated axonal elongation as shown here activates the signaling events needed to trigger changes in the actin and microtubule cytoskeleton required to support axonal formation and axonal elongation (Andersen and Bi, 2000; Bradke and Dotti, 2000). This needs to be tested.

One last result worth discussing in some detail is the axonal regrowth capability of PMGS-overexpressing neurons. It was shown previously that sectioning the axon of a young hippocampal neuron in vitro results in the formation of a new axon from a different neurite if the axonal stump is as long as or shorter than any of the remaining neurites (Goslin and Banker, 1991). We here show that this is indeed the case for control neurons but not for neurons overexpressing PMGS. These neurons consistently form the new axon from the lesioned axon, regardless of the length of the stump (Fig. $6 B, C$ ). The role of PMGS in the regeneration of axons was further supported when we demonstrated that the inhibition of PMGS activity in overexpressing neurons reversed the regenerating effect (Fig. $7 C$ ). One possibility is that the abundant plasma membrane GM1 molecules have established interactions with the extracellular matrix in a number that is more typical of mature axons. This may have prematurely "matured" the overexpressing neuron, which has now fixed axonal and dendritic fate. Such a view is in agreement with our own data showing the premature polarization of the cytoskeletal proteins tau and MAP2 in PMGS-overexpressing neurons.

In addition to the cell biological significance, the lesioning experiments may have a clinical ramification. Because overexpression of PMGS facilitates the regrowth of the original axon and because PMGS mRNA, protein, and enzyme activity levels are very low in the adult hippocampus, one logical assumption is that regeneration of mature neurons could be improved by increasing the activity of PMGS in situ. Patients suffering from GM1 gangliosidosis present defects in ganglioside lysosomal degradation (mutations on the acid $\beta$-galactosidase) with GM1 accumulation, leading to neurodegeneration. The proposed strategy of increasing PMGS activity should not pose such a problem for neuronal systems with a normal lysosomal function, as was the case with our hippocampal cultures. Despite that, testing the effect of PMGS in vivo should rely on the use of inducible elements to allow for an accurate control of enzyme expression.

We are confident that the manipulation of PMGS expression in vivo using novel gene delivery strategies will open new possibilities in nervous system regeneration, an aspect of paramount clinical significance.

\section{REFERENCES}

Andersen SSL, Bi G (2000) Axon formation: a molecular model for the generation of neuronal polarity. BioEssays 22:172-179.

Bradke F, Dotti CG (2000) Establishment of neuronal polarity: lessons from cultured hippocampal neurons. Curr Opin Neurobiol 10:574-581.

Caceres A, Banker G, Steward O, Binder L, Payne M (1984) MAP2 is localized to the dendrites of hippocampal neurons which develop in culture. Brain Res 315:314-318.

Caceres A, Banker GA, Binder L (1986) Immunocytochemical localiza- tion of tubulin and microtubule-associated protein 2 during the development of hippocampal neurons in culture. J Neurosci 6:714-722.

Chaplin MF (1986) Monosaccharides. In: Carbohydrate analysis. A practical approach (Chaplin MF, Kennedy JF eds), pp 1-36. Oxford, UK: IRL.

Craig AM, Banker G (1994) Neuronal polarity. Annu Rev Neurosci 17:267-310

de Hoop MJ, Meyn L, Dotti CG (1998) Culturing hippocampal neurons astrocytes from fetal rodent brain. In: Cell biology: a laboratory handbook (Celis JE, ed), pp 154-163. San Diego: Academic.

Dotti CG, Sullivan CA, Banker GA (1988) The establishment of polarity by hippocampal neurons in culture. J Neurosci 8:1454-1468.

Esch T, Lemmon V, Banker G (1999) Local presentation of substrate molecules directs axon specification by cultured hippocampal neurons. J Neurosci 19:6417-6426.

Facci L, Leon A, Toffano G, Sonnino S, Ghidoni R, Tettamanti G (1984) Promotion of neuritogenesis in mouse neuroblastoma cells by exogenous gangliosides. Relationship between the effect and the cell association of ganglioside GM1. J Neurochem 42:299-305.

Ferrari G, Anderson BL, Stephens RM, Kaplan DR, Greene LA (1995) Prevention of apoptotic neuronal death by GM1 ganglioside. Involvement of Trk neurotrophin receptors. J Biol Chem 270:3074-3080.

Goslin K, Banker G (1991) Rat hippocampal neurons in low-density culture. In: Culturing nerve cells (Banker G, Goslin K, eds) pp 251281. Cambridge, MA: MIT.

Harel R, Futerman AH (1993) Inhibition of sphingolipid synthesis affects axonal outgrowth in cultured hippocampal neurons. J Biol Chem 268:14476-14481.

Hasegawa T, Yamaguchi K, Wada T, Takeda A, Itoyama Y, Miyagi T (2000) Molecular cloning of mouse ganglioside sialidase and its increased expression in Neuro2a cell differentiation. J Biol Chem 275:8007-8015.

Hata K, Wada T, Hasegawa A, Kiso M, Miyagi T (1998) Purification and characterization of a membrane-associated ganglioside sialidase from bovine brain. J Biochem (Tokyo) 123:899-905.

Inokuchi J, Mizutani A, Jimbo M, Usuki S, Yamagishi K, Mochizuki H, Muramoto K, Kobayashi K, Kuroda Y, Iwasaki K, Ohgami Y, Fujiwara M (1997) Up-regulation of ganglioside biosynthesis functional synapse formation memory retention by a synthetic ceramide analog ( LPDMP). Biochem Biophys Res Commun 237:595-600.

Kaether C, Skehel P, Dotti CG (2000) Axonal membrane proteins are transported in distinct carriers: a two-color video microscopy study in cultured hippocampal neurons. Mol Biol Cell 11:1213-1224.

Katoh-Semba R, Skaper SD, Varon S (1984) Interaction of GM1 ganglioside with PC12 pheochromocytoma cells: serum and NGFdependent effects on neuritic growth (and proliferation). J Neurosci Res 12:299-310.

Kopitz J, von Reitzenstein C, Sinz K, Cantz M (1996) Selective ganglioside desialylation in the plasma membrane of human neuroblastoma cells. Gycobiology 6:367-376.

Kopitz J, Muhl C, Ehemann V, Lehmann C, Cantz M (1997) Effects of cell surface ganglioside sialidase inhibition on growth control differentiation of human neuroblastoma cells. Eur J Cell Biol 73:1-9.

Kopitz J, von Reitzenstein C, Burchert M, Cantz M, Gabius HJ (1998) Galectin-1 is a mayor receptor for ganglioside GM1, a product of the growth-controlling activity of a cell surface ganglioside sialidase on human neuroblastoma cells in culture. J Biol Chem 273:11205-11211.

Kozac M (1989) The scanning model for translation: an update. J Cell Biol 108 229-241.

Leeden RW (1989) Biosynthesis metabolism and biological effects of gangliosides. In: Neurobiology of glycoconjugates (Margolis RU, Margolis RK, eds), pp 43-69. New York: Plenum.

Lein PJ, Banker GA, Higgins D (1992) Laminin selectively enhances axonal growth and accelerates the development of polarity by hippocampal neurons in culture. Brain Res Dev Brain Res 69:191-197.

Manthorpe M, Engvall E, Ruoslahti E, Longo GE, Davis GE, Varon S (1983) Laminin promotes neuritic regeneration from cultured peripheral and central neurons. J Cell Biol 97:1882-1890.

Masco D, Van de Walle M, Spiegel S (1991) Interaction of ganglioside GM1 with the B subunit of cholera toxin modulates growth and differentiation of neuroblastoma N18 cells. J Neurosci 11:2443-2452.

McKerracher L, Chamoux M, Arregui CO (1996) Role of laminin and integrin interactions in growth cone guidance. Mol Neurobiol 12:95-116.

Miyagi T, Wada T, Iwamatsu A, Hata K, Yoshikawa Y, Tokuyama S, Sawada M (1999) Molecular cloning and characterization of a plasma membrane-associated sialidase specific for gangliosides. J Biol Chem 274:5004-5011.

Mutoh T, Tokuda A, Guroff G, Fujiki N (1993) The effect of the B subunit of cholera toxin on the action of nerve growth factor on PC12 cells. J Neurochem 60:1540-1547.

Mutoh T, Tokuda A, Miyadai T, Hamaguchi M, Fujiki N (1995) Ganglioside GM1 binds to the Trk protein and regulates receptor function. Proc Natl Acad Sci USA 92:5087-5091.

Nakagami Y, Abe K, Nishiyama N, Matsuki N (2000) Laminin degra- 
dation by plasmin regulates long-term potentiation. J Neurosci 20:2003-2010.

Panni MK, Cooper JD, Sofroniew MV (1998) Ganglioside GM1 potentiates NGF action on axotomised medial septal cholinergic neurons. Brain Res 812:76-80.

Pinkstaff JK, Detterich J, Lynch G, Gall C (1999) Integrin subunit gene expression is regionally differentiated in adult brain. J Neurosci 19:1541-1556.

Pitto M, Mutoh T, Kuriyama M, Ferraretto A, Palestini P, Masserini M (1998) Influence of endogenous GM1 ganglioside on TrkB activity in cultured neurons. FEBS Lett 439:93-96.

Puche AC, Poirier F, Hair M, Bartlett PF, Key B (1996) Role of galectin-1 in the developing mouse olfactory system. Dev Biol 179:274-287.

Quinn CC, Gray GE, Hockfield S (1999) A family of proteins implicated in axon guidance and outgrowth. J Neurobiol 41:158-164.

Rabin SJ, Mocchetti I (1995) GM1 ganglioside activates the high-affinity nerve growth factor receptor trkA. J Neurochem 65:347-354.

Ravichra B, Joshi PG (1999) Regulation of transmembrane signaling by ganglioside GM1: interaction of anti-GM1 with neuro2A cells. J Neurochem 73:557-567.

Reuter G, Schauer R (1994) Determination of sialic acids. Methods Enzymol 230:168-199.

Saito M, Tanaka Y, Tang CP, Yu RK, Ando S (1995) Characterization of sialidase activity in mouse synaptic plasma membranes its agerelated changes. J Neurosci Res 40:401-406.

Schnaar RL, Needham LK (1994) Thin-layer chromatography of glycosphingolipids. Methods Enzymol 230:371-389.

Schwartz M, Spirman N (1982) Sprouting from chicken embryo dorsal root ganglion induced by nerve growth factor is specifically inhibited by affinity purified anti-ganglioside antibodies. Proc Natl Acad Sci USA 79:6080-6083.

Schwarz A, Rapaport E, Hirschberg K, Futerman AH (1995) A regulatory role for sphingolipids in neuronal growth, inhibition of sphingolipid synthesis and degradation have opposite effects on axonal branching. J Biol Chem 270:10990-10998.

Skaper SD, Katoh-Semba L, Varon S (1985) GM1 ganglioside accelerates neurite outgrowth from primary peripheral and central neurons under selected culture conditions. Brain Res 355:19-26.

Spoerri PE, Rapport MM, Mahadik SP, Roisen FJ (1988) Inhibition of conditioned media mediated neuritogenesis of sensory ganglia by monoclonal antibodies to GM1 gangliosides. Dev Brain Res 41:71-77.

Suter DM, Forscher P (1998) An emerging link between cytoskeletal dynamics and cell adhesion molecules in growth cone guidance. Curr Opin Neurobiol 8:106-116.

Tessier-Lavigne M, Goodman CS (1996) The molecular biology of axon guidance. Science 274:1123-1133.

Wada T, Yoshikawa Y, Tokuyama S, Kuwabara M, Akita H, Miyagi T (1999) Cloning, expression and chromosomal mapping of a human ganglioside sialidase. Biochem Biophys Res Commun 261:21-27.

Wang LJ, Colella R, Roisen FJ (1998) Ganglioside GM1 alters neuronal morphology by modulating the association of MAP2 with microtubules and actin filaments. Brain Res Dev Brain Res 105:227-239.

Warren L (1959) The thiobarbituric assay of sialic acids. J Biol Chem 234:1971-1978.

Wu G, Ledeen RW (1991) Stimulation of neurite outgrowth in neuroblastoma cells by neuraminidase: putative role of GM1 ganglioside in differentiation. J Neurochem 56:95-104.

Wu G, Ledeen RW (1994) Gangliosides as modulators of neuronal Ca ${ }^{+}$. Prog Brain Res 101:101-112. 